\title{
Effects of Robot-assisted Gait With Body Weight Support on Torque, Work, and Power of Quadriceps and Hamstring Muscles in Healthy Subjects
}

\author{
Jihun Hwang ${ }^{1}$, PT, MSc, Sung (Joshua) Hyun You², PT, PhD, Woochol Joseph Choi², PT, PhD, Chung-hwi Yi², PT, \\ PhD \\ ${ }^{1}$ Department of Rehabilitation Medicine, Seoul National University Hospital, Seoul, ${ }^{2}$ Department of Physical Therapy, Yonsei University, \\ Wonju, Korea
}

Article Info

Received July 1, 2021

Revised July 12, 2021

Accepted July 13, 2021

Corresponding Author

Chung-hwi Yi

E-mail: pteagle@yonsei.ac.kr

https://orcid.org/0000-0003-2554-8083

\section{Key Words}

Body weight support

Power

Quadriceps and hamstring muscles

Robot-assisted gait training

Torque

Work
Background: Robot-assisted gait training (RAGT) is an effective method for walking rehabilitation. Additionally, the body weight support (BWS) system reduces muscle fatigue while walking. However, no previous studies have investigated the effects of RAGT with BWS on isokinetic strength of quadriceps and hamstring muscles.

Objects: The purpose of this study was to investigate the effects of torque, work, and power on the quadriceps and hamstring muscles during RAGT, using the BWS of three conditions in healthy subjects. The three different BWS conditions were BWS 50\%, BWS $20 \%$, and full weight bearing (FWB).

Methods: Eleven healthy subjects ( 7 males and 4 females) participated in this study. The Walkbot_S was used to cause fatigue of the quadriceps and hamstring muscles and the Biodex Systems 4 Pro was used to measure the isokinetic torque, work, and power of them. After RAGT trials of each of the three conditions, the subjects performed isokinetic concentric knee flexion and extension, five at an angular velocity of $60 \% \mathrm{~s}$ and fifteen at an angular velocity of $180 \%$. One-way repeated analysis of variance was used to determine significant differences in all the variables. The least significant difference test was used for post-hoc analysis.

Results: On both sides, there were significant differences in peak torque (PT) of knee extension and flexion between the three BWS conditions at an angular velocity of $60 \% \mathrm{~s}$ and $180 \% \mathrm{~s}$ conditions. A post-hoc comparison revealed that the PT in the BWS $50 \%$ was significantly greater than in the BWS 20\% and the FWB and the PT in the BWS 20\% was significantly greater than in the FWB.

Conclusion: The results of this study suggest that the lower BWS during RAGT seems to lower the isokinetic torque, work, and power of the quadriceps and hamstring muscles because of the muscle fatigue increase.

\section{INTRODUCTION}

Robot-assisted gait training (RAGT) is a training method that has reduced the limitations of conventional physiotherapy and gait training with body weight support (BWS) on the treadmill. RAGT can be used as a new treatment for people who have difficulty with walking independently and it is effective for improving functional walking ability and muscle activity. Based on the theory of repetitive task specific training for the re-learning of walking, RAGT is performed by combining BWS treadmill training with mechanical exoskeletal devices, which provide an automated gait training [1,2]. The RAGT system is designed to induce locomotor movements with appropriate leg muscle activation according to a program based on a normal physiological gait pattern that is pre-inputted. In particular, RAGT has the advantage of delivering consistently reproducible, guided movement for the lower extremities [3]. This robotic device is used in combination with BWS systems to unload the partial body weight of the subject during training so as to reduce the burden on the lower limbs [4,5]. Therefore, this training allows the patient to perform a normal gait by supporting their weight and reducing the load [6]. Besides, RAGT is used for isokinetic strength training, which can affect muscle strength, power, and endurance depending on weight 
support loading.

Isokinetic muscle strength is the work that is performed within a given time when the force is exerted on the device and it also means its tension in terms of torque or newtonmeter [7]. Peak torque (PT) is the ability to generate force at low speed and it is commonly measured at an angular speed of 60 degrees; work and power are the ability to generate force at high speed and are commonly measured at an angular speed of 180 degrees [8]. This method often used in measuring concentric contraction fatigue in quadriceps or hamstrings, for example, involves assessing the decline in maximum torque or work values over a predetermined number of exercise repetitions at a constant intermediated angular velocity of 180 degrees [9]. The percentage of decline in PT production during both concentric contraction of the quadriceps was examined by Gray and Chandler (1989) [10]. They defined isokinetic fatigue as the percentage decline in PT from the initial value to the final value. Depending on the isokinetic measurement protocols, the percentage decline in the PT during concentric contraction of quadriceps ranged from $12 \%$ to $55 \%$ at high angular velocities [10-13] and from $6 \%$ to $35 \%$ at low angular velocities [11,14-16].

Despite many studies [17-22] having been conducted for RAGT, it has not yet been demonstrated with clear standards and guidelines. During the robot rehabilitation training, it is important to consider various conditions such as BWS, speed, and resistance. To train subjects with various physical conditions, it is necessary to analyze and confirm the dynamic mechanical characteristics and normal gait patterns of subjects. However, there has been no clear evidence regarding how walking speed, stride length, BWS and the range of motion (ROM) affect the subjects' gait pattern during the RAGT. In particular, inappropriate BWS during RAGT can place an excessive load on the hip, knee and ankle joints, which will easily lead to pathological gait after long-term training [23-25]. Thus, the purpose of this study was to investigate the effects of isokinetic torque, work, and power on the quadriceps and hamstring muscles during the RAGT, using the BWS of three conditions (BWS 50\%, BWS 20\%, and full weight bearing [FWB]) in healthy subjects. The hypothesis of this study was that in healthy subjects the isokinetic parameters of the knee extensor and flexor would be different among the three BWS conditions during RAGT.

\section{MATERIALS AND METHODS}

\section{Subjects}

Eleven healthy subjects (7 males, 4 females; age: $26.7 \pm 3.5$ years, height: $171.9 \pm 8.3 \mathrm{~cm}$, weight: $66.1 \pm 9.2 \mathrm{~kg}$, body mass index [BMI]: $13.9 \pm 5.2 \mathrm{~kg} / \mathrm{m}^{2}$ ) were recruited from the Department of Rehabilitation Medicine at Seoul National University hospital in South Korea (Table 1). The inclusion criteria included: 1) aged from 18 to 40 years, 2) weighing less than $100 \mathrm{~kg}$, 3) with a height of more than $140 \mathrm{~cm}$ and less than $200 \mathrm{~cm}$, and 4) a body mass index of less than $25 \mathrm{~kg} / \mathrm{m}^{2}$. The exclusion criteria included the following: 1) lower limb injury within the last six months, 2) tightness or limited ROM with the knee for maximal and minimal ROM of the experimental device, and 3) past or present neurological, musculoskeletal, and cardiopulmonary diseases.

The experimental protocols and purpose of this study were explained in detail to all participants; they subsequently provided written informed consent. This study was approved by the Seoul National University Hospital Institutional Review Board (H-1703-122-843).

\section{Experimental Apparatus}

The study involved the use of a Walkbot_S (Walkbot_S, P \& S Mechanics Co. Ltd., Seoul, Korea) for the RAGT having been widely used in the rehabilitation. A Biodex System 4 Pro (Biodex Medical Systems Inc., Shirley, USA) was used to assess the isokinetic strength of the knee extension and flexion. The data generated during maximal isokinetic contraction were recorded to a computer-linked Biodex. An Inbody 770 (Biospace Inc., Seoul, Korea) using bioelectrical impedance analysis was used to measure the BMI. The classifications are as follows: (Normal range: 18.5-24.9, Overweight: 25-29.9, Obesity: 30-39.9, severe obesity: $\geq 40$ )

Table 1. General characteristics of the subjects $(N=11)$

\begin{tabular}{|c|c|c|}
\hline & $\begin{array}{c}\text { Mean } \pm \text { standard } \\
\text { deviation }\end{array}$ & Range \\
\hline Age (y) & $26.7 \pm 3.5$ & $22-32$ \\
\hline Height $(\mathrm{cm})$ & $171.9 \pm 8.3$ & $159-187.9$ \\
\hline Weight (kg) & $66.1 \pm 9.2$ & $51.4-80.3$ \\
\hline Body fat mass (kg) & $22.3 \pm 1.6$ & $20.3-25.0$ \\
\hline Body mass index $\left(\mathrm{kg} / \mathrm{m}^{2}\right)$ & $13.9 \pm 5.2$ & $6.9-21.7$ \\
\hline Sex (male/female) & \multicolumn{2}{|c|}{$7 / 4$} \\
\hline Dominant side (right/left) & \multicolumn{2}{|c|}{$11 / 0$} \\
\hline
\end{tabular}




\section{Experimental Procedure}

\section{1) Study design}

Three BWS conditions included the following: 1) BWS 50\%, 2) BWS 20\%, and 3) FWB. The order of each condition was randomly assigned using a table of random numbers. In this study, the RAGT was performed once a day for 30 minutes to avoid muscle fatigue after training. The subjects were measured by isokinetic evaluation at two angular velocities immediately after 30 minutes of RAGT for each condition. Three RAGTs and three isokinetic evaluations were performed for three days.

\section{2) Robot-assisted gait training}

The subjects were positioned in such a way that their hip and knee joint axes were closely aligned to the hip and knee of the Walkbot_S. The screen would provide biofeedback information such as torque, speed, time, steps, and distance. The subjects were actively trained while maintaining a constant torque value through the screen display. The treadmill walking speed was selected as $1.5 \mathrm{~km} / \mathrm{h}$ which was used in previous studies on robot walking and was gradually increased to 2.3 $\mathrm{km} / \mathrm{h}$ as soon as possible in accordance with the most comfortable gait for each subject [26]. A harness was used to support the weight of the subjects on the treadmill. The BWS was $0 \%, 20 \%$, and $50 \%$ of body weight, and hands were positioned on the handrail for balance. Before the RAGT trials, a 5-minute warm-up exercise was performed. To equalize the subject's effort during RAGT for each condition, an active movement was performed while maintaining a constant torque value using the biofeedback that appeared on the monitor screen (Figure 1). The training was performed for 30 minutes, which is the treatment time that is commonly used in clinics.

\section{3) Isokinetic testing}

Before the isokinetic trials, the dynamometer was calibrated, and the ROM was set from $0^{\circ}$ to $90^{\circ}$ for knee flexion and $90^{\circ}$ to $0^{\circ}$ for knee extension. The trunk, pelvis and thigh of each subject was fixed by using a velcro strap to minimize any unnecessary compensation movement during the taking of the measurement. The rotational axis of the knee was aligned with the rotational axis of the dynamometer based on the position of the tibial epicondyle. The pad, which is a control accessory, was placed $5 \mathrm{~cm}$ above the lateral malleolus of the ankle. The subjects performed the maximal concentric contraction for knee flexion and extension in the sitting position (Figure 2). Both legs were tested and the participants' dominant side was always tested first. For isokinetic testing, the participants performed five maximal contractions of the knee flexion and extension at a velocity of $60 \%$ s and then fifteen maximal contractions at a velocity of $180^{\circ} / \mathrm{s}$ [27]. The subjects were allowed a 20 -second rest between the angular velocity conditions.

\section{Statistical Analysis}

The Statistical Package for the Social Sciences (Windows ver. 23.0; IBM Co., Armonk, NY, USA) was used for all statistical analyses. A one-sample Kolmogorov-Smirnov test was used to confirm the normality of all the subjects. A one-way re-

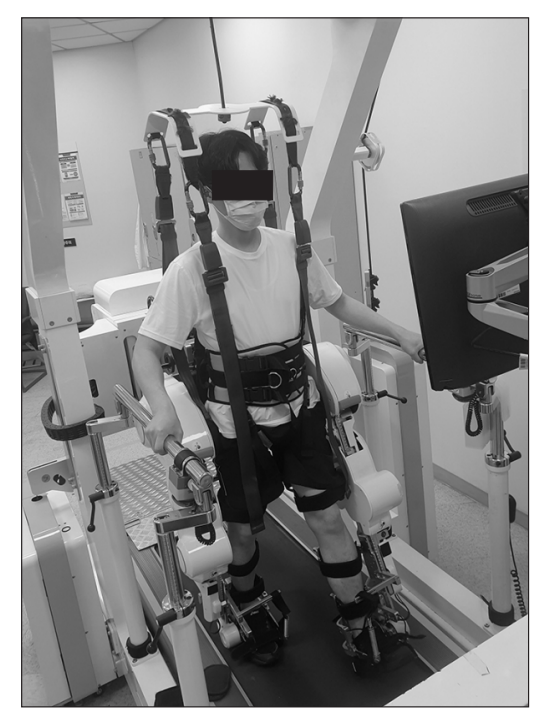

Figure 1. Robot-assisted gait training.

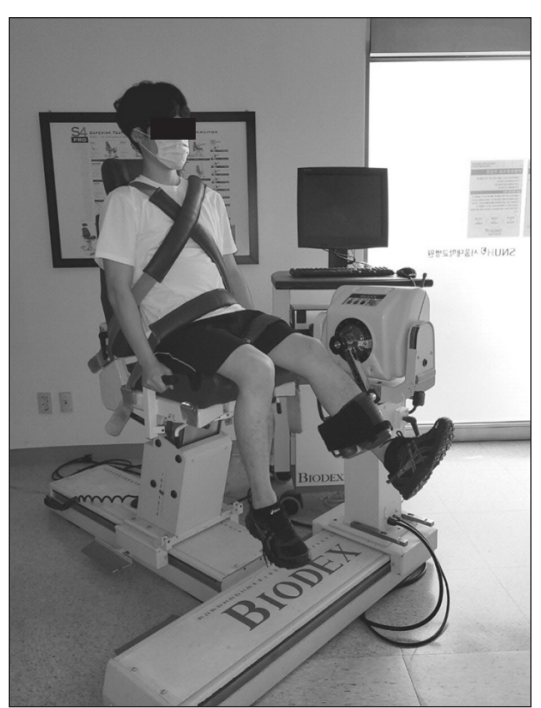

Figure 2. Isokinetic testing. 
Table 2. Isokinetic knee extension at an angular velocity 60 degrees

\begin{tabular}{clccccc}
\hline & & BWS 50\% & BWS 20\% & FWB & F-value & p-value \\
\hline Right extension & PT (Nm) & $169.12 \pm 48.77$ & $160.75 \pm 45.95$ & $145.12 \pm 42.13$ & 36.97 & $<0.01^{* *}$ \\
(dominant) & TW (J) & $602.52 \pm 192.55$ & $564.94 \pm 146.45$ & $508.55 \pm 156.84$ & 13.89 & $<0.01^{* *}$ \\
& AP (W) & $102.71 \pm 35.61$ & $93.98 \pm 28.17$ & $88.27 \pm 27.26$ & 12.18 & $<0.01^{* *}$ \\
Left extension & PT (Nm) & $165.24 \pm 50.88$ & $155.39 \pm 45.38$ & $142.04 \pm 44.05$ & 37.92 & $<0.01^{* *}$ \\
& TW (J) & $588.85 \pm 179.03$ & $562.52 \pm 154.94$ & $493.00 \pm 147.30$ & 8.71 & $<0.01^{* *}$ \\
& AP (W) & $103.05 \pm 33.68$ & $92.58 \pm 32.04$ & $86.44 \pm 26.94$ & 15.78 & $<0.01^{* *}$ \\
\hline
\end{tabular}

Values are presented as mean \pm standard deviation. BWS, body weight support; FWB, full weight bearing; PT, peak torque; Nm, Newton meter; TW, total work; AP, average power. ${ }^{* *} p<0.01$.

Table 3. Isokinetic knee extension at an angular velocity 180 degrees

\begin{tabular}{clccrrr}
\hline & & BWS $50 \%$ & BWS 20\% & FWB & F-value & p-value \\
\hline Right extension & PT (Nm) & $105.94 \pm 29.09$ & $94.98 \pm 25.08$ & $85.43 \pm 24.19$ & 62.03 & $<0.01^{* *}$ \\
(dominant) & TW (J) & $1,247.98 \pm 350.16$ & $1,093.19 \pm 296.17$ & $1,031.28 \pm 321.87$ & 7.72 & $<0.01^{* *}$ \\
& AP $(W)$ & $149.87 \pm 43.23$ & $129.15 \pm 35.98$ & $123.78 \pm 39.34$ & 13.87 & $<0.01^{* *}$ \\
Left extension & PT (Nm) & $103.92 \pm 29.18$ & $96.00 \pm 27.73$ & $82.13 \pm 21.64$ & 35.46 & $<0.01^{* *}$ \\
& TW (J) & $1,212.01 \pm 309.25$ & $1,115.51 \pm 303.94$ & $938.95 \pm 243.89$ & 23.88 & $<0.01^{* *}$ \\
& AP (W) & $146.08 \pm 38.70$ & $132.41 \pm 37.03$ & $112.79 \pm 31.44$ & 36.63 & $<0.01^{* *}$ \\
\hline
\end{tabular}

Values are presented as mean \pm standard deviation. BWS, body weight support; FWB, full weight bearing; PT, peak torque; Nm, Newton meter; TW, total work; AP, average power. ${ }^{* *} p<0.01$.
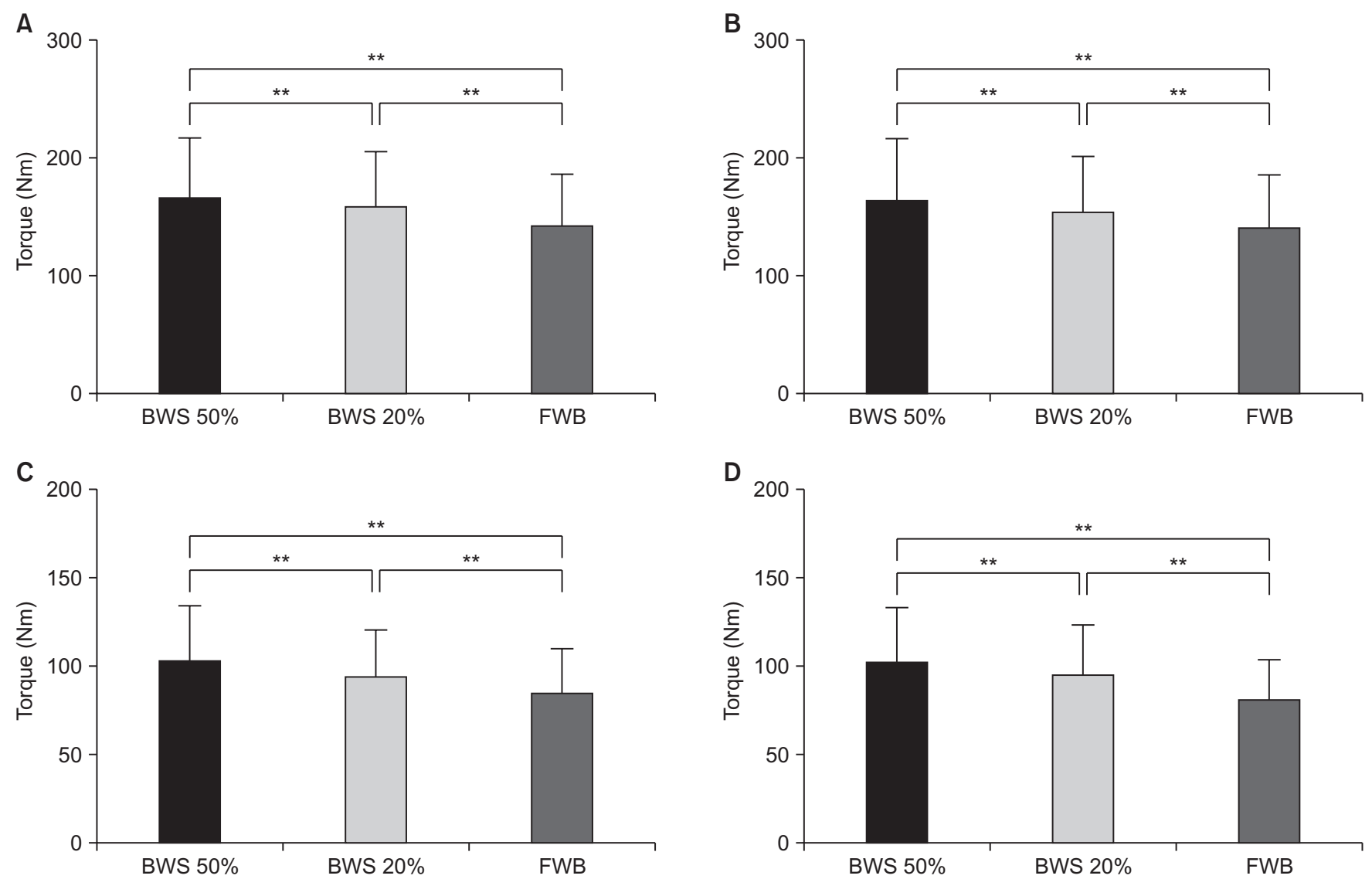

Figure 3. Peak torque of knee extension in the three conditions. (A) Right extension. (B) Left extension at an angular velocity of $60 \%$ s. (C) Right extension. (D) Left extension at an angular velocity of $180 \%$ s. Nm, Newton meter; BWS, body weight support; FWB, full weight bearing. Error bar: standard deviation. ${ }^{*} \mathrm{p}$ $<0.05,{ }^{* *} p<0.01$. 
peated measures analysis of variance was used to compare the isokinetic strength of the knee flexor and the extensor under three conditions. The Mauchly's test was used to determine whether or not they met the sphericity assumption. When the sphericity assumption was not met but the correction was possible, the Greenhouse-Geisser value was presented. The least significant difference test was used for post- hoc analysis. A pvalue of less than 0.05 was considered statistically significant for all analyses.

\section{RESULTS}

\section{Isokinetic Knee Extension at an Angular Velocity of 60 and 180 Degrees}

On the both side, the PT, total work (TW), and average power (AP) of knee extension revealed significant differences between the three types of RAGT at an angular velocity of $60 \%$ and $180^{\circ}$ / s ( $\left.<0.05\right)$ (Tables 2, 3). The post-hoc comparison results were presented in the Figures 3-5.
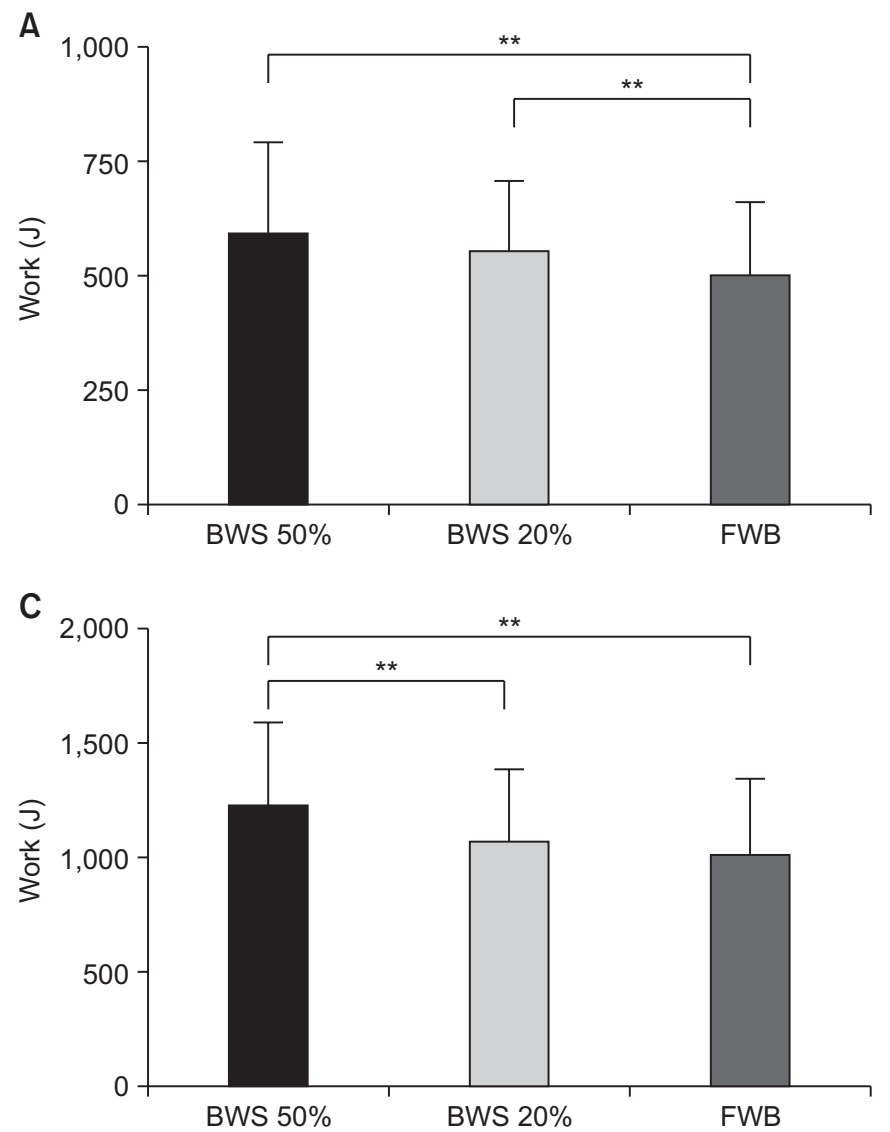

\section{Isokinetic Knee Flexion at an Angular Velocity of 60 and 180 Degrees}

On the both side, the PT, TW, and AP of knee flexion revealed significant differences between the three types of RAGT at an angular velocity of $60 \%$ s and $180 \%$ s $<0.05)$ (Tables 4 , 5). The post-hoc comparison results were presented in the Figures 6-8.

\section{DISCUSSION}

The results of this study show that the lower the BWS was during RAGT the lower the isokinetic torque, work, and power of quadriceps and hamstring muscles became. After RAGT, the isokinetic strength of the quadriceps and hamstring muscles was significantly different between the three BWS conditions at both angular velocities of 60 and $180 \%$ s. A decrease in the recorded isokinetic muscle strength in the knee joint was generally accompanied by muscle fatigue in healthy subjects, as illustrated in the example shown in Figures 3-8. The PT of the knee joints in the BWS 50\% was significantly greater than in
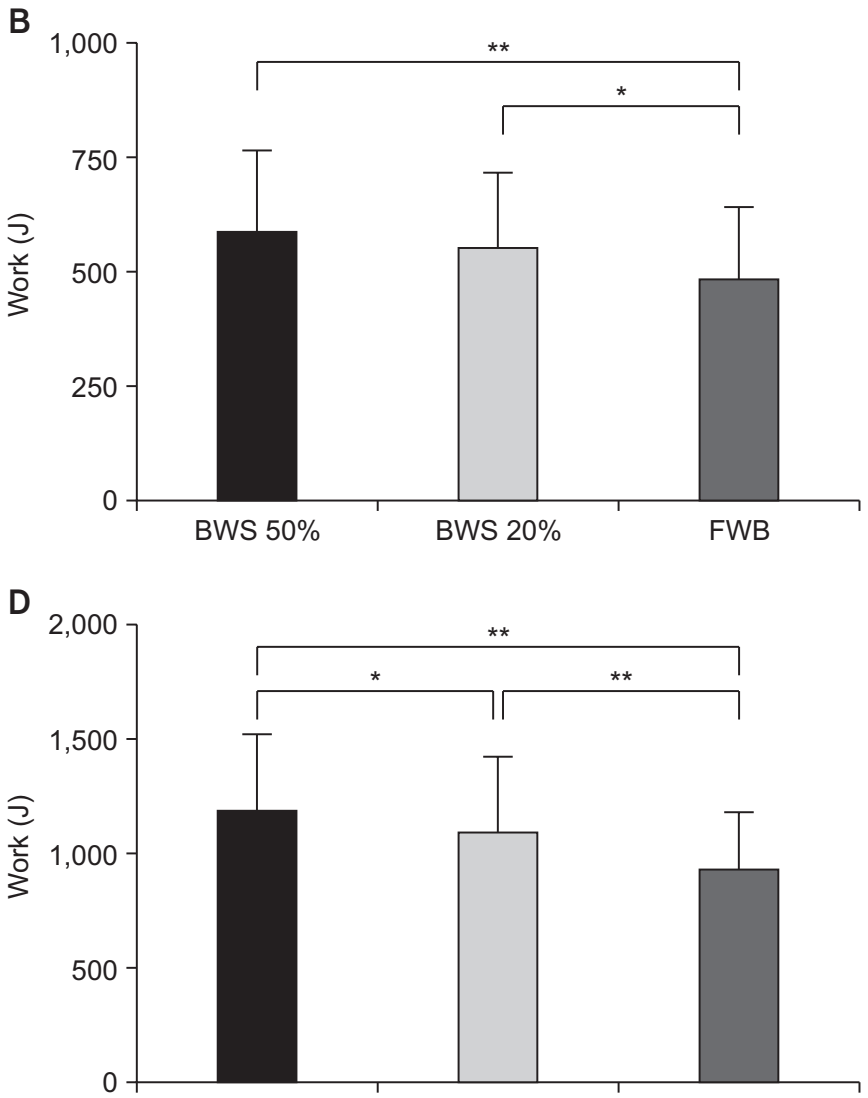

BWS $50 \%$

BWS $20 \%$

FWB

Figure 4. Total work of knee extension in the three conditions. (A) Right extension. (B) Left extension at an angular velocity of $60 \%$ s. (C) Right extension. (D) Left extension at an angular velocity of $180 \%$ s. BWS, body weight support; FWB, full weight bearing. Error bar: standard deviation. ${ }^{*} p<0.05,{ }^{* *} p<0.01$. 

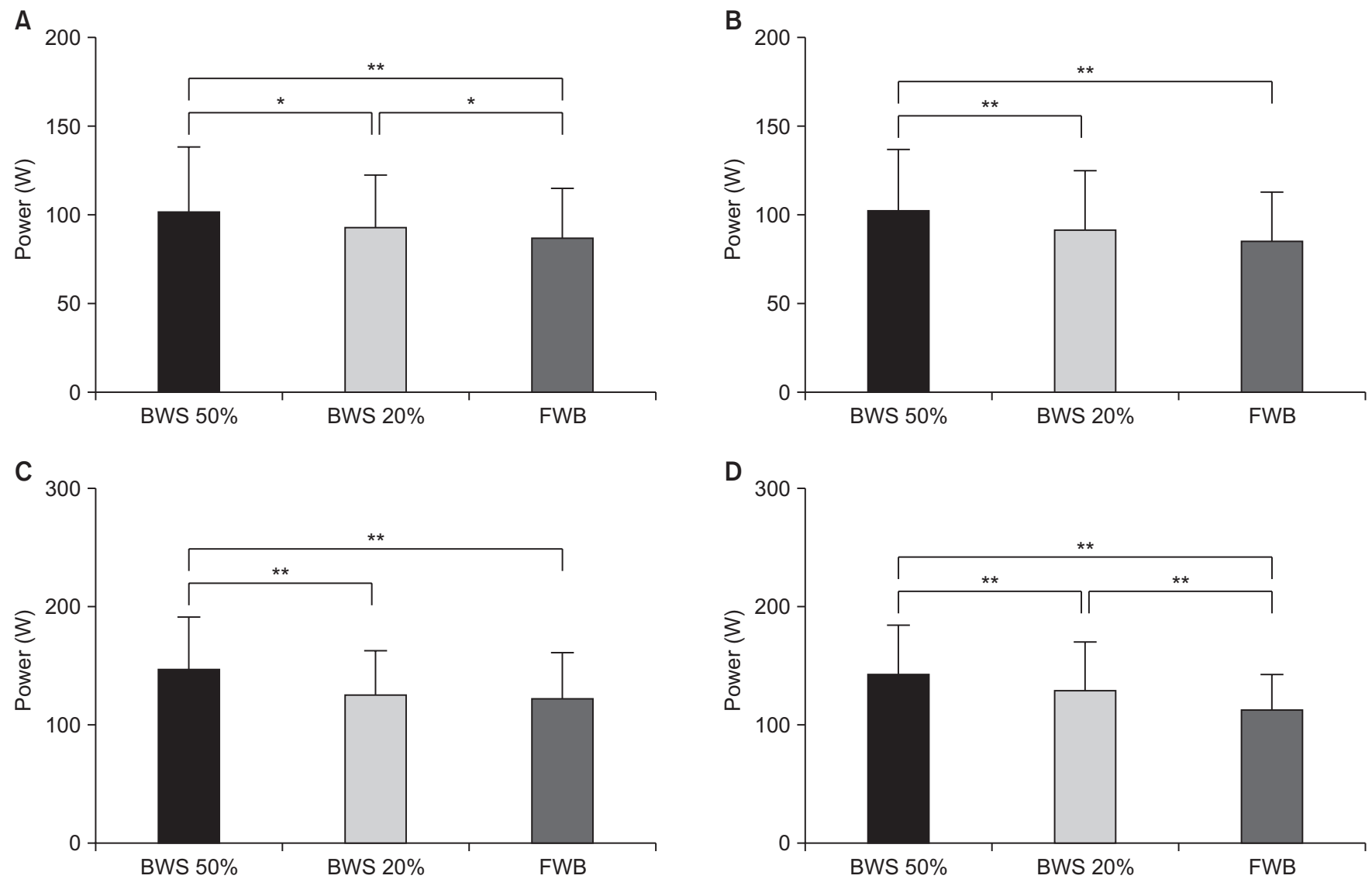

Figure 5. Average power of knee extension in the three conditions. (A) Right extension. (B) Left extension at an angular velocity of $60 \%$ s. (C) Right extension. (D) Left extension at an angular velocity of $180 \%$ s. BWS, body weight support; FWB, full weight bearing. Error bar: standard deviation. ${ }^{*} p<0.05,{ }^{* *} p$ $<0.01$.

Table 4. Isokinetic knee flexion at an angular velocity 60 degrees

\begin{tabular}{|c|c|c|c|c|c|c|}
\hline & & BWS 50\% & BWS 20\% & FWB & F-value & $\mathrm{p}$-value \\
\hline \multirow{3}{*}{$\begin{array}{l}\text { Right extension } \\
\text { (dominant) }\end{array}$} & $\mathrm{PT}(\mathrm{Nm})$ & $92.58 \pm 32.26$ & $83.89 \pm 29.20$ & $77.08 \pm 27.33$ & 14.57 & $<0.01^{* *}$ \\
\hline & TW (J) & $364.68 \pm 146.63$ & $355.17 \pm 127.22$ & $309.28 \pm 148.52$ & 5.65 & $<0.05^{*}$ \\
\hline & AP $(W)$ & $59.35 \pm 26.04$ & $56.22 \pm 22.78$ & $50.86 \pm 23.21$ & 4.81 & $<0.05^{*}$ \\
\hline \multirow[t]{3}{*}{ Left extension } & $\mathrm{PT}(\mathrm{Nm})$ & $86.45 \pm 28.99$ & $75.44 \pm 25.82$ & $70.35 \pm 26.15$ & 20.21 & $<0.01^{* *}$ \\
\hline & TW (J) & $337.02 \pm 152.80$ & $319.12 \pm 139.44$ & $269.79 \pm 124.81$ & 10.54 & $<0.01^{* *}$ \\
\hline & $\mathrm{AP}(\mathrm{W})$ & $55.89 \pm 26.95$ & $56.73 \pm 32.28$ & $43.86 \pm 20.96$ & 6.05 & $<0.01^{* *}$ \\
\hline
\end{tabular}

Values are presented as mean \pm standard deviation. BWS, body weight support; FWB, full weight bearing; PT, peak torque; Nm, Newton meter; TW, total work; AP, average power. ${ }^{*} p<0.05,{ }^{* *} p<0.01$.

Table 5. Isokinetic knee flexion at an angular velocity 180 degrees

\begin{tabular}{clccccc}
\hline & & BWS $50 \%$ & BWS 20\% & FWB & F-value & p-value \\
\hline Right extension & PT (Nm) & $67.74 \pm 21.65$ & $59.48 \pm 18.92$ & $54.08 \pm 17.00$ & 27.80 & $<0.01^{* *}$ \\
(dominant) & TW (J) & $776.35 \pm 401.37$ & $654.08 \pm 309.50$ & $560.81 \pm 224.61$ & 6.91 & $<0.01^{* *}$ \\
& AP (W) & $86.49 \pm 44.28$ & $71.38 \pm 32.78$ & $63.08 \pm 24.94$ & 7.01 & $<0.01 * *$ \\
Left extension & PT (Nm) & $62.58 \pm 21.64$ & $55.68 \pm 17.14$ & $49.96 \pm 13.72$ & 13.15 & $<0.01^{* *}$ \\
& TW (J) & $748.21 \pm 377.40$ & $612.58 \pm 295.19$ & $494.08 \pm 219.78$ & 15.04 & $<0.01^{* *}$ \\
& AP (W) & $82.48 \pm 39.95$ & $67.03 \pm 30.96$ & $55.05 \pm 25.81$ & 17.48 & $<0.01 * *$ \\
\hline
\end{tabular}

Values are presented as mean \pm standard deviation. BWS, body weight support; FWB, full weight bearing; PT, peak torque; Nm, Newton meter; TW, total work; AP, average power. ${ }^{* *} p<0.01$. 

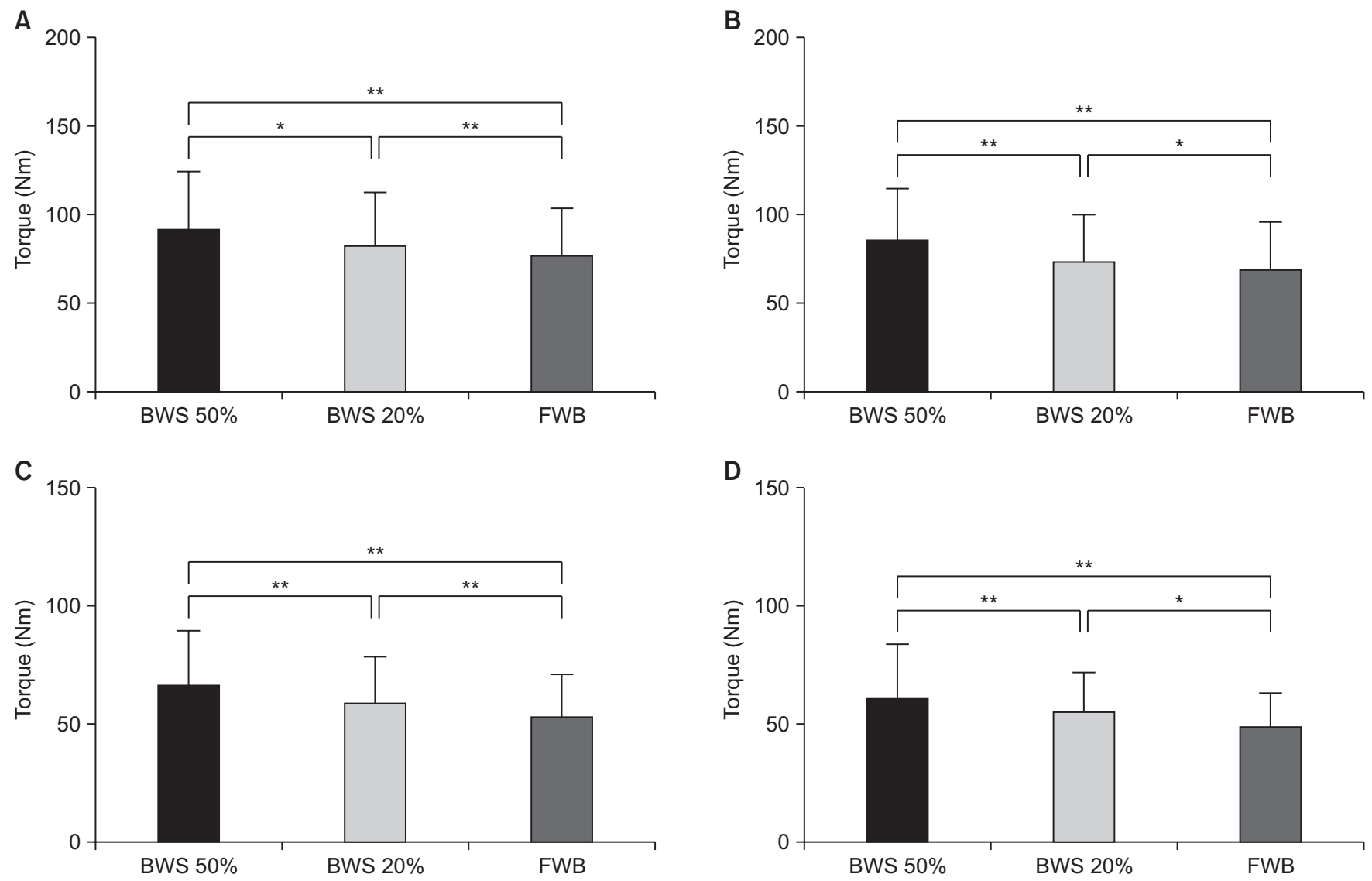

Figure 6. Peak torque of knee flexion in the three conditions. (A) Right flexion. (B) Left flexion at an angular velocity of $60 \%$ s. (C) Right flexion. (D) Left flexion at an angular velocity of $180 \%$ s. Nm, Newton meter; BWS, body weight support; FWB, full weight bearing. Error bar: standard deviation. ${ }^{*} p<0.05,{ }^{* *} p$ $<0.01$.

the BWS 20\% and the FWB, the PT in BWS the $20 \%$ was significantly greater than in the FWB. To the best of my knowledge, no previous studies have examined the isokinetic fatigue during RAGT with three BWS conditions. Therefore, it is impossible to compare the results of the current study to other studies. The results of the present study indicate that the higher BWS that affects the lower extremities when there is contact with the ground showed a smaller decrease in muscle strength. This means that the load on the legs during walking is reduced, which lowers resistance for the movements that are required to make walking. The ground reaction force is minimized in the mid-stance phase, because the center of gravity (COG) matched the body alignment against the gravity line and body weight was unloaded [28]. In particular, the COG height becomes higher, the higher the potential energy, and the higher the energy efficiency. Conversely, if the potential energy is lowered in the double stance phase, more kinetic energy must be used. Therefore, it could be considered that less fatigue occurs because of reduced muscle effort. When walking on the ground in FWB, the body vector is located at the front of the hip joint and the knee joint is located at the back in the heel strike of the stance phase [29]. Also, the forefoot and posterior foot pressure are higher than in the middle foot, which is because the weight load occurred mainly in the forefoot and the posterior foot in the initial swing phase and the loading response of the stance phase [30]. As BWS increases, the extension moment is provided to suppress the weight load on both the hip and the knee joints and the foot pressure reduces significantly, which reduces the overall pressure of the ankle joints. The reduction of forefoot pressure in the initial swing phase makes it easier to perform knee flexion and ankle plantarflexion. As a result, the hamstring muscles and the gastrocnemius activity are reduced depending on the BWS increase. In addition, an eccentric contraction of quadriceps muscles occurs during the loading response of the stance phase, absorbing the shock on the ground, so quadriceps muscle activity is reduced due to posterior foot pressure reduction, depending on the BWS increase [29]. A study showed that quadriceps and 

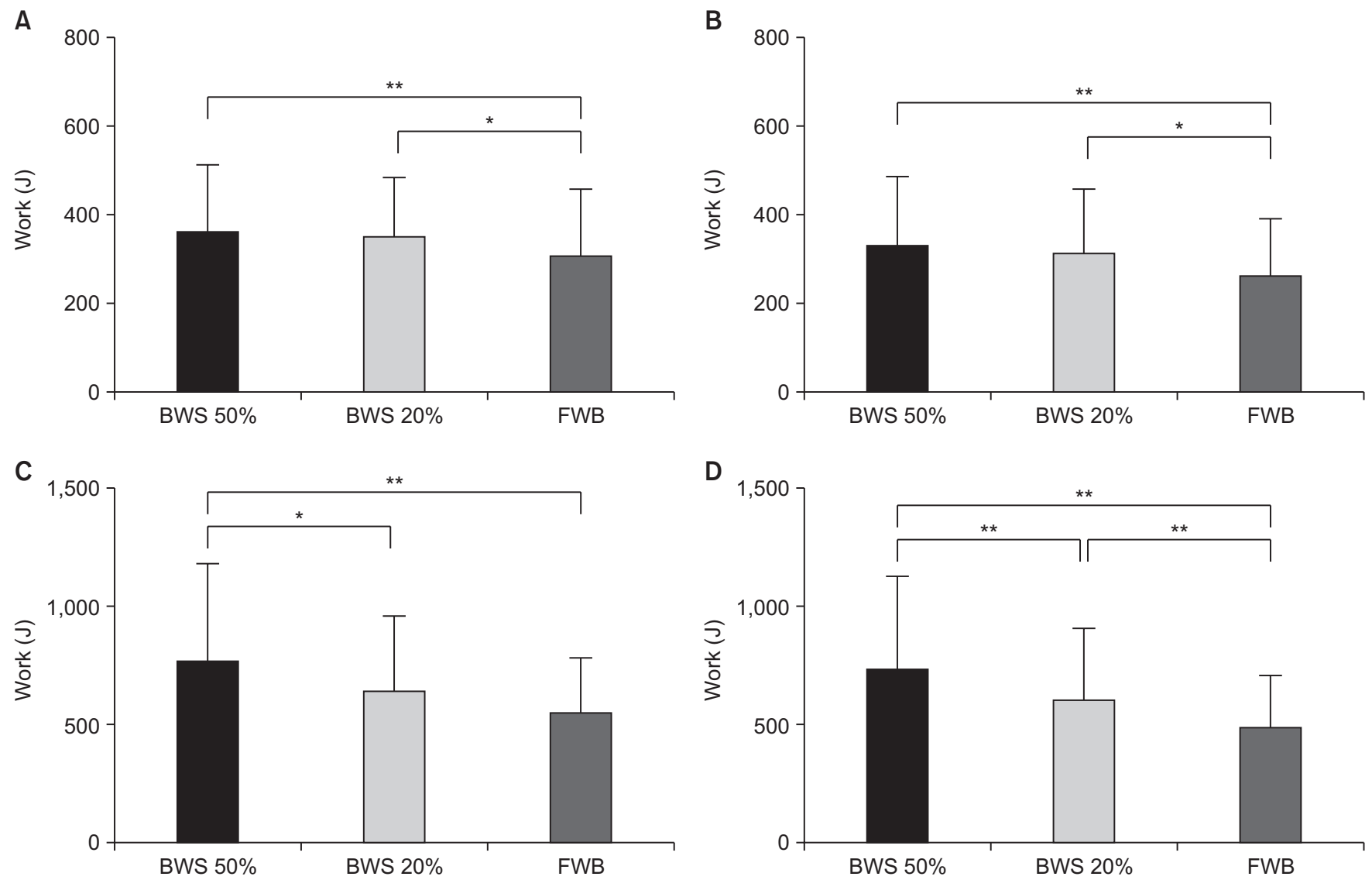

Figure 7. Total work of knee flexion in the three conditions. (A) Right flexion. (B) Left flexion at an angular velocity of $60 \%$ s. (C) Right flexion. (D) Left flexion at an angular velocity of $180 \%$ s. BWS, body weight support; FWB, full weight bearing. Error bar: standard deviation. ${ }^{*} p<0.05,{ }^{* *} p<0.01$.

hamstring muscle activities in BWS 40\% during walking were lowered more significantly than in BWS 0\% [31]. These results are similar to those in this study in that the lower the BWS, the higher the muscle fatigue due to an increase in knee muscle effort.

A key feature of RAGT is the possibility of generating supportive torques and providing guidance of leg movements by the exoskeleton [32]. The RAGT in this study was used as one method of isokinetic exercise in healthy subjects. There has already been an efficient standardized program of isokinetic exercise formats. This is recommended by a level of 50\% muscle fatigue. This fatigue concept, which represents a decrease from the initial value to the final value, is not new and this clinician has experienced a high level of success when applying the following procedures to subject exercise regimens [3335]. Besides this, it may be applied to isokinetic exercise at any joint in either the velocity spectrum or the speed-specific formats [36]. In order to investigate the amount of PT change depending on BWS in this study, the BWS 20\% and BWS 50\% conditions were set as the initial values, respectively, and the
FWB condition was set as a final value. The amount of PT change was as follows. Compared to FWB on the right knee extension at an angular velocity of $60 \%$, BWS $20 \%$ decreased by $9.72 \%$ and BWS $50 \%$ decreased by $14.19 \%$. On the left side, BWS 20\% decreased by $8.59 \%$ and BWS 50\% decreased by $14.04 \%$. On the right knee extension at an angular velocity of $180 \%$, BWS $20 \%$ decreased by $10.05 \%$ and BWS $50 \%$ decreased by $19.36 \%$. On the left side, BWS $20 \%$ decreased by $14.45 \%$ and BWS $50 \%$ decreased by $20.97 \%$. Compared to FWB on the right knee flexion at an angular velocity of $60 \%$ s, BWS $20 \%$ decreased by $8.12 \%$ and BWS $50 \%$ decreased by $16.74 \%$. On the left side, BWS 20\% decreased by $6.75 \%$ and BWS 50\% decreased by $18.62 \%$. On the right knee flexion at an angular velocity of $180 \%$ s, BWS $20 \%$ decreased by $9.08 \%$ and BWS $50 \%$ decreased by $20.17 \%$. On the left side, BWS $20 \%$ decreased by $10.27 \%$ and BWS $50 \%$ decreased by $20.17 \%$. These results are similar to the findings of other studies $[11,15,16]$ on isokinetic strength of knee extensors. For example, Denadai et al. [14] reported that the isokinetic PT of the knee extensors decreased after a session of high intensity exhaustive running exercise. The 
A

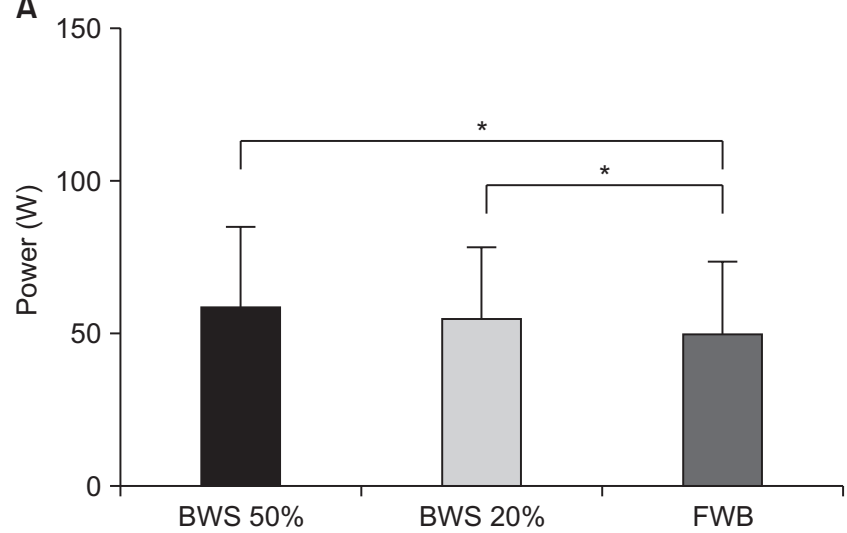

C

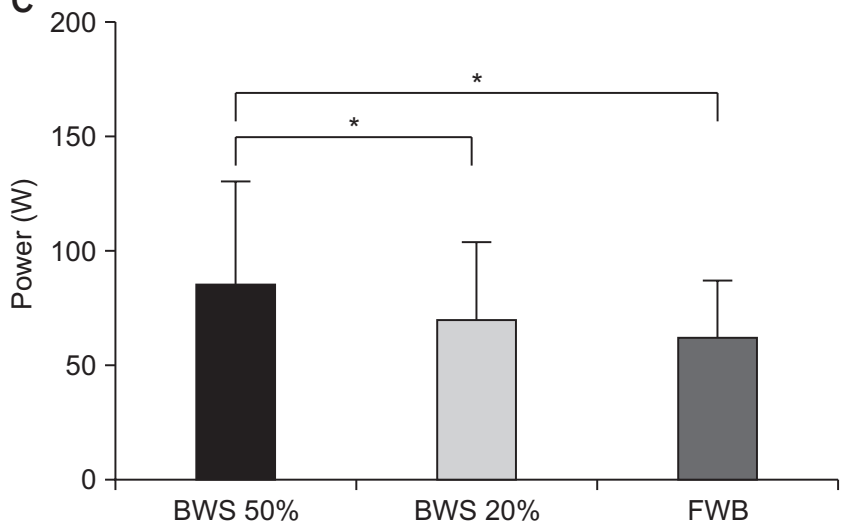

B

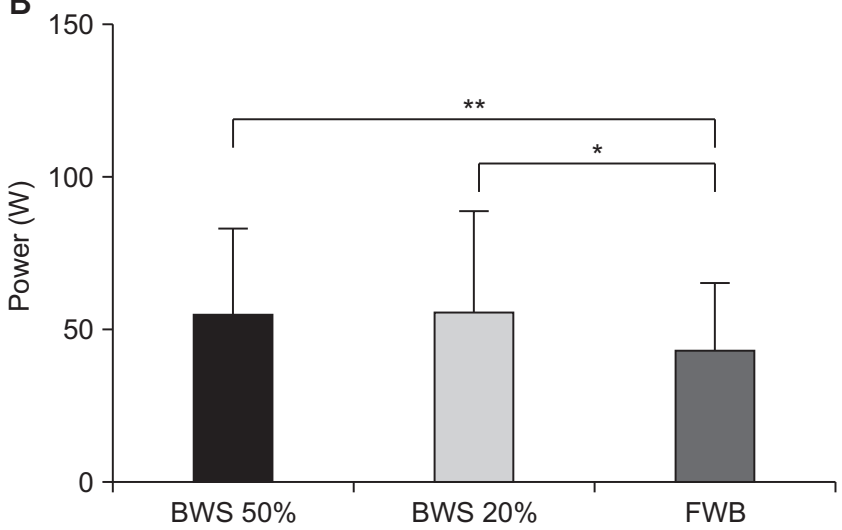

D

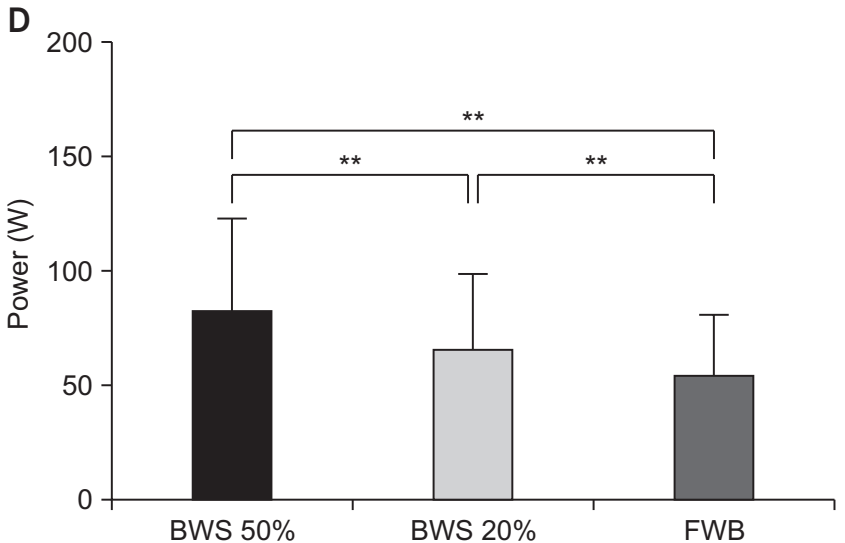

Figure 8. Average power of knee flexion in the three conditions. (A) Right flexion. (B) Left flexion at an angular velocity of $60 \%$ s. (C) Right flexion. (D) Left flexion at an angular velocity of $180 \%$ s. BWS, body weight support; FWB, full weight bearing. Error bar: standard deviation. ${ }^{*} p<0.05,{ }^{* *} p<0.01$.

isokinetic strength losses in our study were higher than the findings, which were founded by Denadai et al. [14] who have reported decreases of $6.5 \%$ and $3.3 \%$ in concentric contraction at $60 \%$ and $180 \%$, respectively. The isokinetic exercise used in this study is a high intensity exercise than running exercise used in their study [14]. Thus, our data indicated that this isokinetic exercise requires a more muscle strength than running. Although RAGT used as an isokinetic fatigue test does not decrease to $50 \%$ fatigue, quantitative data have been presented on how much torque falls when applying BWS. Based on this concept, this data will serve as a useful basis for clinicians using RAGT in the future.

In this study, four isokinetic parameters were recorded: PT, TW, and AP. There are very strong correlations between the PT and TW [37]. In addition, due to the characteristics of the moment curve, there is a close relationship between the PT and TW. This very strong relationship has been studied and reported before [38]. Moreover, as the power derives directly from the work, the other two are also closely related. These relationships are evident from Figures 3-8, which depict very similar variations in magnitude irrespective of the parameter, relative to the experimental condition. The use of the PT seems perfectly sufficient for assessing the isokinetic strength of muscles [39]. Thus, the PT value was explained as the isokinetic muscle strength because all the variables (PT, TW, and AP) showed a similar tendency.

For a purposeful exploitation of the training parameters in RAGT, it is essential to understand how parameter settings affect locomotor control and human body characteristics [40]. This study shows that the lower BWS during RAGT lowered the isokinetic torque, work, and power of the knee muscles because of muscle fatigue increase. However, this study has several limitations. First, the results of this study are representative of a young, healthy population, which may not be generalizable to other populations. Second, the sEMG measurements were not measured to confirm the fatigue of the knee muscles during the isokinetic trials. However, since sEMG amplitude and isokinetic strength were correlated, the isokinetic trial was performed instead of sEMG. Third, the change in foot pressure was not measured according to the change rate of 
BWS through the harness. Finally, the baseline isokinetic test was not measured prior to performing RAGT. The RAGT with the two BWS conditions in this study were set as the baseline values, respectively, because the application of BWS was investigated rather than focusing just on the RAGT data. Further studies are needed to assess sEMG and foot pressure. Besides, the baseline value of isokinetic test is needed to investigate only the fatigue of RAGT.

\section{CONCLUSIONS}

The results of this study suggest that the lower BWS during RAGT seems to lower the isokinetic torque, work, and power of the quadriceps and hamstring muscles because of the muscle fatigue increase. This change in isokinetic muscle strength (PT, TW, and AP) generated by knee joints that exceeds the baseline measures, may be used as an indicator that helps the clinician to continue training or to alter the rehabilitation strategy. Finally, this quantitative data can be used as evidence of what constitutes the correct amount by which to increase or decrease the load when applying BWS during RAGT in clinical practice because the BWS of RAGT requires proper adjustment to the subjects' characteristics and conditions.

\section{ACKNOWLEDGEMENTS}

This study was supported by the "Brain Korea 21 FOUR Project”, the Korean Research Foundation for Department of Physical Therapy in the Graduate School of Yonsei University.

\section{CONFLICTS OF INTEREST}

No potential conflict of interest relevant to this article was reported.

\section{AUTHOR CONTRIBUTIONS}

Conceptualization: JH, CY. Data curation: JH. Formal analysis: JH, CY. Investigation: JH. Methodology: JH, SHY, WC, CY. Project administration: JH, SHY, WC, CY. Resources: JH, SHY, WC, CY. Software: JH. Supervision: JH, SHY, WC, CY. Validation: JH, CY. Visualization: JH. Writing - original draft: JH. Writing - review \& editing: JH, SHY, WC, CY.

\section{ORCID}

Jihun Hwang, https://orcid.org/0000-0002-7371-3840

Sung (Joshua) Hyun You, https://orcid.org/0000-0002-1608-9287

Woochol Joseph Choi, https://orcid.org/0000-0002-6623-3806

\section{REFERENCES}

1. Colombo G, Joerg M, Schreier R, Dietz V. Treadmill training of paraplegic patients using a robotic orthosis. J Rehabil Res Dev 2000;37(6):693-700.

2. Jezernik S, Colombo G, Keller T, Frueh H, Morari M. Robotic orthosis lokomat: a rehabilitation and research tool. Neuromodulation 2003;6(2):108-15.

3. Lo AC, Triche EW. Improving gait in multiple sclerosis using robot-assisted, body weight supported treadmill training. Neurorehabil Neural Repair 2008;22(6):661-71.

4. Pietrusinski M, Cajigas I, Severini G, Bonato P, Mavroidis C. Robotic gait rehabilitation trainer. IEEE ASME Trans Mechatron 2014;19(2):490-9.

5. Lu Q, Liang J, Qiao B, Ma O. A new active body weight support system capable of virtually offloading partial body mass. IEEE ASME Trans Mechatron 2013;18(1):11-20.

6. Barbeau H, Visintin M. Optimal outcomes obtained with bodyweight support combined with treadmill training in stroke subjects. Arch Phys Med Rehabil 2003;84(10):1458-65.

7. Sienna PA. One rep max: a guide to beginning weight training. Indianapolis: Benchmark Press; 1989.

8. Davies RA. Isolated-joint testing \& exercise: a handbook for using Cybex 770 and the U.B.X.T. Bay Shore: Lumec; 1985.

9. Davies GJ. A compendium of isokinetics in clinical usage and rehabilitation techniques. Onalaska: S \& S Publishers; 1992.

10. Gray JC, Chandler JM. Percent decline in peak torque production during repeated concentric and eccentric contractions of the quadriceps femoris muscle. J Orthop Sports Phys Ther 1989;10(8):309-14.

11. Lepers R, Pousson ML, Maffiuletti NA, Martin A, Van Hoecke J. The effects of a prolonged running exercise on strength characteristics. Int J Sports Med 2000;21(4):275-80.

12. Nilsson J, Tesch P, Thorstensson A. Fatigue and EMG of repeated fast voluntary contractions in man. Acta Physiol Scand 1977;101(2):194-8.

13. Thorstensson A, Karlsson J. Fatiguability and fibre composition of human skeletal muscle. Acta Physiol Scand 1976; 
98(3):318-22.

14. Denadai BS, Greco CC, Tufik S, de Mello MT. Effects of high intensity running to fatigue on isokinetic muscular strength in endurance athletes. Isokinetics and Exercise Science 2007;15(4):281-5.

15. Glace BW, McHugh MP, Gleim GW. Effects of a 2-hour run on metabolic economy and lower extremity strength in men and women. J Orthop Sports Phys Ther 1998;27(3):189-96.

16. Sherman WM, Armstrong LE, Murray TM, Hagerman FC, Costill DL, Staron RC, et al. Effect of a 42.2-km footrace and subsequent rest or exercise on muscular strength and work capacity. J Appl Physiol Respir Environ Exerc Physiol 1984;57(6):1668-73.

17. Banala SK, Kim SH, Agrawal SK, Scholz JP. Robot assisted gait training with active leg exoskeleton (ALEX). IEEE Trans Neural Syst Rehabil Eng 2009;17(1):2-8

18. Beyl P, Van Damme M, Van Ham R, Vanderborght B, Lefeber D. Design and control of a lower limb exoskeleton for robotassisted gait training. Applied Bionics and Biomechanics 2009;6(2):229-43.

19. Cao J, Xie SQ, Das R, Zhu GL. Control strategies for effective robot assisted gait rehabilitation: the state of art and future prospects. Med Eng Phys 2014;36(12):1555-66.

20. Hussain S, Xie SQ, Liu G. Robot assisted treadmill training: mechanisms and training strategies. Med Eng Phys 2011; 33(5):527-33.

21. Koenig A, Omlin X, Zimmerli L, Sapa M, Krewer C, Bolliger M, et al. Psychological state estimation from physiological recordings during robot-assisted gait rehabilitation. J Rehabil Res Dev 2011;48(4):367-85

22. Nakanishi Y, Wada F, Saeki S, Hachisuka K. Rapid changes in arousal states of healthy volunteers during robot-assisted gait training: a quantitative time-series electroencephalography study. J Neuroeng Rehabil 2014;11:59.

23. Gordon KE, Ferris DP, Roberton M, Beres JA, Harkema SJ. The importance of using an appropriate body weight support system in locomotor training. Soc Neurosci 2000;26(1):160.

24. Frey M, Colombo G, Vaglio M, Bucher R, Jörg M, Riener R. A novel mechatronic body weight support system. IEEE Trans Neural Syst Rehabil Eng 2006;14(3):311-21.

25. Franz JR, Glauser M, Riley PO, Della Croce U, Newton F, Allaire PE, et al. Physiological modulation of gait variables by an active partial body weight support system. J Biomech 2007;40(14):3244-50.
26. Morone G, Bragoni M, Iosa M, De Angelis D, Venturiero V, Coiro P, et al. Who may benefit from robotic-assisted gait training? A randomized clinical trial in patients with subacute stroke. Neurorehabil Neural Repair 2011;25(7):636-44.

27. Bonetti LV, Grisa NC, Demeda CS, Finger ALT, De Marchi T, Tadiello GS. Isokinetic performance of knee extensor and flexor musculature in adolescent female handball players. Arch Med Deporte 2018;35(3):157-61.

28. Qin T, Zhang L. Coordinated control strategy for roboticassisted gait training with partial body weight support. J Cent South Univ 2015;22(8):2954-62.

29. Perry J, Burnfield JM. Gait analysis: normal and pathological function. 2nd ed. Thorofare: Slack; 2010.

30. Hessert MJ, Vyas M, Leach J, Hu K, Lipsitz LA, Novak V. Foot pressure distribution during walking in young and old adults. BMC Geriatr 2005;5:8.

31. Soucy MT. Examining the effects of body weight support and speed on physiological measures and lower leg muscular activity. Las Vegas, University of Nevada, Master's Thesis. 2016.

32. Riener R, Lünenburger L, Maier IC, Colombo G, Dietz V. Locomotor training in subjects with sensori-motor deficits: an overview of the robotic gait orthosis lokomat. J Healthc Eng 2010;1(2):197-216.

33. Sherman WM, Pearson DR, Plyley MJ, Costill DL, Habansky AJ, Vogelgesang DA. Isokinetic rehabilitation after surgery. A review of factors which are important for developing physiotherapeutic techniques after knee surgery. Am J Sports Med 1982;10(3):155-61.

34. Sherman WM, Plyley MJ, Pearson DR, Habansky AJ, Vogelgesang DA, Costill DL. Isokinetic rehabilitation after meniscectomy: a comparison of two methods of training. Physician Sportsmed 1983;11(4):121-33.

35. Smith MJ, Melton P. Isokinetic versus isotonic variable-resistance training. Am J Sports Med 1981;9(4):275-9.

36. Timm KE. Isokinetic exercise to 50\% fatigue. J Orthop Sports Phys Ther 1987;8(10):505-6.

37. Dvir Z, David G. Average or peak moment: which of the two is more suitable to represent isokinetic muscle strength? Isokinet Exerc Sci 1995;5(2):93-7.

38. Dvir Z. Isokinetics: muscle testing, interpretation, and clinical applications. 2nd ed. Edinburgh: Churchill Livingstone; 2004.

39. Hwang JH, Sung KS, Yi CH. Effects of abdominal hollowing and bracing maneuvers on hip extension strength in prone standing position. Isokinet Exerc Sci 2020;28(2):161-9. 
40. Van Kammen K, Boonstra A, Reinders-Messelink H, den Otter R. The combined effects of body weight support and gait speed on gait related muscle activity: a comparison between walk- ing in the Lokomat exoskeleton and regular treadmill walking. PLoS One 2014;9(9):e107323. 